\title{
SwissDRG: Bereits zwei Jahre vergangen ...
}

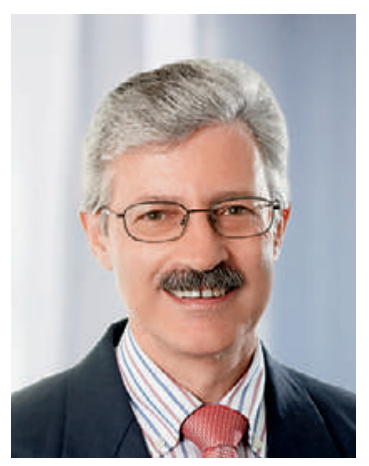

Die seit dem 1.Januar 2012 angewendete Tarifstruktur SwissDRG liegt bereits in der dritten Version vor. Obwohl sich der Alltag der stationär behandelten Patienten seitdem nicht wesentlich geändert hat, stehen die Leitungen der medizinischen Einrichtungen nunmehr vor zahlreichen Schwierigkeiten. So sind von einer Version zur anderen in bestimmten Bereichen grosse Unterschiede bei den Kostengewichten festzustellen. Andererseits hat die - wenn auch moderate - Erhöhung der Anzahl der Zusatzentgelte die Versicherer dazu veranlasst, eine mögliche Kostensteigerung durch die jährlich ausgehandelte Senkung der Baserates vorwegzunehmen. Das System ist also noch sehr labil, was eine vorausschauende Planung schwierig macht.

Der Einschluss der Investitionen und Anlagenutzungskosten in die SwissDRG-Struktur bereitet weiterhin Probleme. Die bisherige normative Lösung ist nicht zufriedenstellend, da der festgelegte Prozentsatz nicht den realen Bedarf abdeckt. Angesichts dieses Mangels schränken bestimmte Einrichtungen ihren Betrieb ein, um investieren zu können. Andere wiederum werden von ihrem Kanton unterstützt, der sie durch gemeinwirtschaftliche Leistungen mitunter grosszügig subventioniert. Aufgrund der wachsenden Budgetzwänge ist diese Verzerrung jedoch rückläufig.

\section{Die Konkurrenz nimmt sowohl zwischen öffentlichen Spitälern und privaten Einrichtungen als auch in der Rekrutierung der Ärztinnen und Ärzte zu.}

Im Hinblick auf die Verhaltensweisen wurde bei den Leistungserbringern keine offenkundige Kursänderung festgestellt, abgesehen von einer leichten Tendenz zu fragmentierten Aufenthalten von Patientinnen und Patienten, die mehrere Pathologien gleichzeitig aufweisen. Die Versicherer hingegen sperren sich deutlich gegen das Erteilen von Kostengutsprachen in der Rehabilitation. Da diese erst nach Ausschöpfung der normalen Aufenthaltsdauer in akutsomatischen Spitälern gewährt werden, werden effizient arbeitende Spitäler bestraft.

Die nationale Spitallandschaft hat sich noch nicht auffällig verändert, erste Tendenzen zeichnen sich aber bereits ab. Einige private Einrichtungen haben ausgebaut, insbesondere in den Gebieten, die durch die Auswirkungen der Rationalisierung des öffentlichen Spitalnetzes aufgegeben wurden. Der Grund: Jetzt können sie vom staatlichen Anteil profitieren und eine strenge Risikoauswahl praktizieren. Aufgrund des Mangels an qualifizierten Fachkräften in bestimmten Disziplinen führt dies zu einer starken Konkurrenz in der Rekrutierung der Ärztinnen und Ärzte, da eine beträchtliche Anzahl unserer Kolleginnen und Kollegen die öffentlichen Spitäler verlässt und ihre Patienten in die Kliniken mitnimmt.

\section{Der Ausgleich zwischen Regulierung und Konkurrenz muss ständig \\ neu bewertet werden.}

Was beschert uns die Zukunft? Vieles hängt davon ab, inwieweit die Tarifstruktur ausreichend verfeinert werden kann, damit sie die Spitalleistungen noch besser abbildet. Das Weiterbestehen von differenzierten Baserates nach Spitalkategorie bleibt offen. Derzeit werden die grossen nicht universitären Zentralspitäler benachteiligt, da sie nicht von einem höheren Multiplikationsfaktor wie die Universitätsspitäler profitieren. Die Ausübung der hochspezialisierten Medizin in mittelgrossen Einrichtungen könnte für diese Zentren ebenfalls eine Vorzugsrate rechtfertigen, wie es die SUVA durch eine tarifliche Differenzierung der Trauma Centers anerkennt.

Grundsätzlich sind die Einführung des SwissDRG und die Änderungen bei der Spitalfinanzierung starke Antriebskräfte für die Entwicklung. Die schnellen Wege der Patientenversorgung und die Vereinfachungsprotokolle setzen sich zunehmend durch. Andererseits werden teure Geräte, die keinen wissenschaftlich nachweisbaren Mehrwert bringen, zu Recht in Frage gestellt. Ohne dass die technischen Fortschritte verleugnet werden, ist hier eine gewisse Rückkehr zum gesunden Menschenverstand zu beobachten.

Die aktuelle Entwicklung des Spitalwesens ist zweifellos spannend. Der Ausgleich zwischen Regulierung und Konkurrenz muss ständig neu bewertet werden. Wie dieser Gegensatz künftig aufgelöst wird, wirkt sich massgeblich auf das Land aus. Aus diesem Grund engagiert sich die FMH entschlossen in dieser Debatte, damit unsere humanistische Vision zum Wohle der Patientinnen und Patienten berücksichtigt und sichergestellt wird.

Dr. Pierre-François Cuénoud, Vizepräsident der FMH, Departementsverantwortlicher Tarife und Gesundheitsökonomie Spitalärzte 